\title{
Shape, sizing optimization and material selection based on mixed variables and genetic algorithm
}

\author{
Xingang Tang • David Hicham Bassir • \\ Weihong Zhang
}

Received: 3 November 2008 / Accepted: 24 August 2010 / Published online: 25 September 2010

(C) The Author(s) 2010. This article is published with open access at Springerlink.com

\begin{abstract}
In this work, we explore simultaneous designs of materials selection and structural optimization. As the material selection turns out to be a discrete process that finds the optimal distribution of materials over the design domain, it cannot be performed with common gradient-based optimization methods. In this paper, material selection is considered together with the shape and sizing optimization in a framework of multiobjective optimization of tracking the Pareto curve. The idea of mixed variables is often introduced in the case of mono-objective optimization. However, in the case of multi-objective optimization, we still face some hard key points related to the convexity and the continuity of the Pareto domain, which underline the originality of this work. In addition to the above aspect, there is a lack in the literature concerning the industrial applications that consider the mixed parameters. Continuous variables refer to structural parameters such as thickness, diameter and spring elastic constants while material ID is defined as binary design variable for each material. Both mechanical and thermal loads are considered in this work with the aim of minimizing the maximum stress and structural weight simultaneously. The efficiency of the design procedure is demonstrated through various numerical examples.
\end{abstract}

\footnotetext{
X.G. Tang · W.H. Zhang

Engineering Simulation and Aerospace Computing (ESAC), The Key Laboratory of Contemporary Design \& Integrated Manufacturing Technology, Northwestern Polytechnical University,

710072 Xi'an, Shaanxi, China

e-mail: nwputang@yahoo.com.cn

W.H. Zhang

e-mail: zhangwh@nwpu.edu.cn

D.H. Bassir ( $₫)$

Aerospace Structures, Faculty of Aerospace Engineering, Delft University of Technology,

2629 HS Delft, The Netherlands

e-mail: D.H.Bassir@tudelft.nl

D.H. Bassir

FIT/ESTP/Constructability, Research Institute, 28, Avenue Président Wilson, 94234 Cachan, France
} 


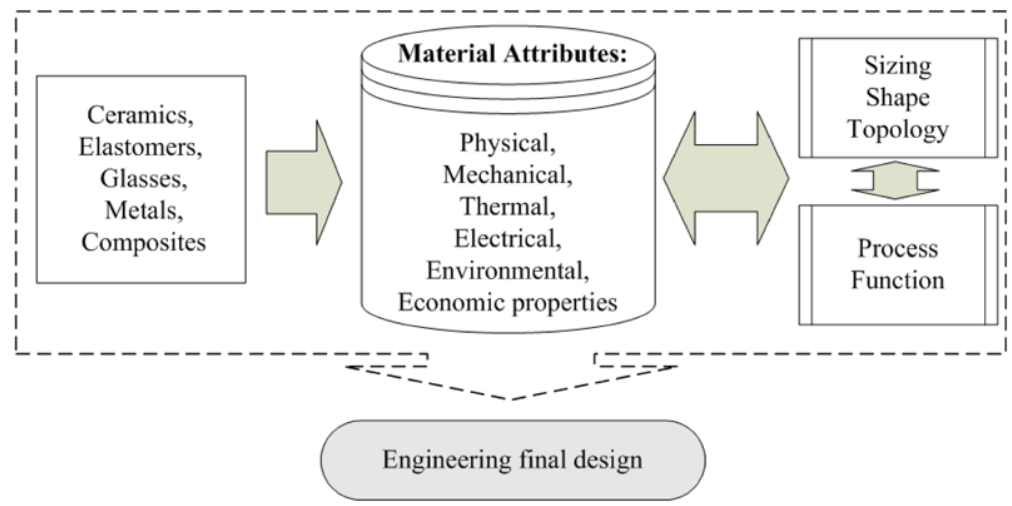

Fig. 1 Relevant interactions between function, material, geometry and manufacturing process with key decision-making in the conception process

Keywords Material selection · Shape optimization · Mixed design variables · Multiobjective optimization · Genetic algorithms

\section{Introduction}

It is well understood that material selection plays an important role in engineering design (Tawancy et al. 2007; Alexopoulos 2007; Matos and Simplio 2006; Guisbiers and Wautelet 2007; Deng and Edwards 2007; Edwards 2005). However, materials are generally regarded as the physical attributes of a mechanical system. They are often considered after the conceptual design stage, such as in detail design (Walker and Smith 2002; Edwards and Deng 2007; Fredricson 2005). Although the structure is thus validated in its size and geometry, the final design has to meet with the requirements that highly depend on the materials used (Fig. 1). Therefore, the identifications of appropriate combinations of materials have to be performed and working principles have to be followed judiciously. In other words, the design task is to compare the properties of a finite set of materials and select the best one out of this finite set, i.e., materials selection or material identification. Compared with other conceptual design procedures such as topology, shape or sizing optimization (Bendsoe et al. 1994; Jung and Gea 2006; Zhang et al. 1999, 2007, 2008), material identification is more critical and it represents about $70 \%$ of the product life cycle cost. However, this stage receives relatively less tool and technique supports in the design process.

In the engineering design community, size, shape, and topology optimization procedures are three classes of extensively studied design methodologies with assumed materials (Bendsoe et al. 1994; Zhang et al. 1999; Torstenfelt and Klarbring 2007). These optimization procedures can be either performed one by one or in an all-inone multicriteria optimization manner. In the latest literature, no work has introduced the idea of simultaneous optimization including the material selection under thermomechanical loading. This is due to the fact that gradient-based algorithms are mainly limited to continuous variables (Guisbiers and Wautelet 2007; Walker and Smith 2002, 2006; Fredricson 2005; Bendsoe et al. 1994; Walker et al. 1997; Rakshit and 
Ananthasuresh 2008; Kumar and Singh 2007; Ghoreishi et al. 2007) and graph theory is limited only for discrete variables (Rao 2006). Rakshit (2008) considered the simultaneous optimization of geometry and material selection of statically determinate trusses as a continuous problem. Bendsoe (1994) used the topology optimization method to determine the structure topology as well as the material microstructure of desired properties. In his strategy, the micro parameters involved in the constitutive stress-strain relationship are taken as design variables together with the macro topology variables in the optimization problem. The advantage is that different material properties at each point are determined in the optimized topology. But a material with such optimized properties may not exist in reality (Hörnlein and Kocvara 2001). Furthermore, having different microstructures for different parts of the structure is relatively challenging with the current state of manufacturing facilities (Edwards 2005; Edwards and Deng 2007). Although the material selection can be done either before or after the structure is optimized, an optimal match between the structure and materials cannot be guaranteed. Therefore, designers should consider structural optimization and material selection simultaneously. The corresponding problem may lead to multicriteria or multiobjective optimization where the Pareto curve may be discontinuous and not convex.

In this paper, a short description of the heuristic GA approach (Guessasma and Bassir 2010a, 2010b; Bassir et al. 2007, 2009b; Deb 2007; Goldberg 1989; Michalewicz 1994; Deb et al. 2002; Holland 1975) is firstly presented. The algorithm considers the double coding aspects: discrete and continuous to allow the exploration of simultaneous geometry design and material selection for the structures. The efficiency of the proposed approach will be demonstrated through one mathematical function and two mechanical examples of plate and beam structures.

\section{Optimization with mixed variables}

Because of the mixed nature of the material and geometry optimization, we need an efficient algorithm that considers the discrete choice of the material selection and the continuous choice of the geometry profile. On the one hand, the latter can be performed by means of gradient-based methods once the sensitivity analysis is easily carried out (Zhang et al. 1999). On the other hand, some combinatory algorithms can be used (Rao 2006) when only discrete variables are involved. Here, the work is firstly focused upon the pure discrete problems for which the genetic algorithm is applied through the binary coding. Secondly, we will describe the binary coding and the real coding by using a modified version of the NSGA-II (Bassir et al. 2007; Deb et al. 2002) program for the multiobjective optimization of mixed problems.

\subsection{Genetic algorithm}

Based on Darwinian survival-of-the best fitness principal that mimics natural biological evolution, Genetic algorithms (GAs) provide an alternative of traditional optimization techniques to locate the optimal solutions in a complex landscape. The theoretical foundations were first led by Holland in 1975 (Holland 1975) 
Fig. 2 Principal function of a simple genetic algorithm

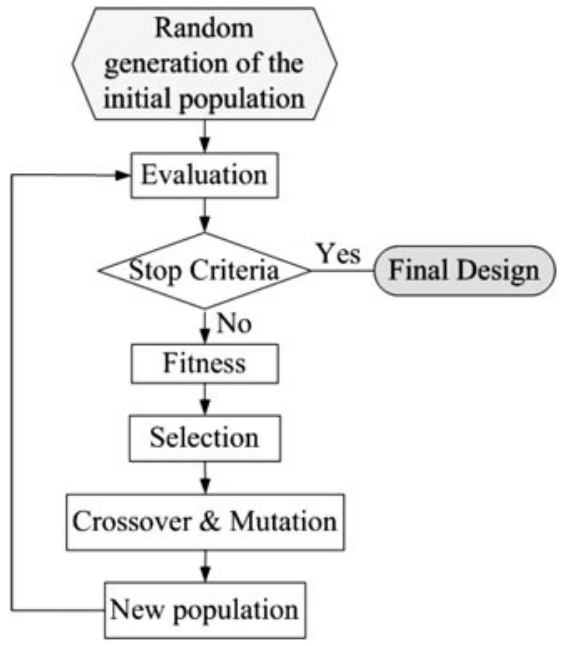

and since that time, the number of applications and publications concerning GAs has increased exponentially. In 1989, Goldberg gave signs of nobility to GAs as an efficient and general method to treat complex optimization problems (Goldberg 1989). The principle of GAs described in Fig. 2 is to simulate the evolution of one population of individuals (randomly chosen) to which we apply different production operators (selection, crossover and mutation). As GAs start the searching from different initial solutions, a global solution of the problem is expected (Guessasma and Bassir 2010a, 2010b; Bassir et al. 2007, 2009b; Deb 2007; Goldberg 1989). This global perspective prevents GAs from being trapped locally and allows them to explore the overall design domain. Besides, such algorithms deal with the problem only by virtue of the function values. They can be implemented as a black box with several entries and one exit (Guessasma and Bassir 2010a).

It is largely demonstrated that the choice of the operators and the representation of the design parameters are very important in the optimization process.

Three main points are involved when using genetic algorithms: the first point is the feature of the application: single or multiobjective optimization, the second point is the design space (discrete, continuous or mixed domain) and the third point is the constraints handling.

In the case of multiobjective optimization, the operator that performs the selection is no more the same, because some solutions are not "comparable" any more in terms of criterion but rather in terms of ranking. The dominance relation that relates two solutions is therefore a binary operator. In general, the result of this operation for two individual solutions in the criteria space has two possibilities: either one solution dominates another or the solutions do not dominate each other. For one giving point in objective function space, it could be dominated or not dominated by another point, but it could also be "incomparable or non-dominated" to other points (Bassir et al. 2009a, 2009b; Irisarri et al. 2009). Regarding the generation and selection of the Pareto optimal set, an ordering technique (sorting process) is required. When using an evolutionary algorithm for generating such Pareto optimal set. Thus, various ranking methods have been suggested in the specialized literature. Such methods essentially 
sort the individuals in objective function space before selection. Each member of the list of possible points in objective function space is assigned a rank. The result of the dominance ranking is a strict partial ordered list which is used for sorting the points before employing a desired selection operator. To maintain the diversity of the population, these classified individuals are shared with their dummy fitness values. Then this group of classified individuals is ignored and another layer of non-dominated individuals is considered. The process continues until all individuals or a part of the population is classified. Since individuals in the first front have the maximum fitness value, they always get more copies than the rest of the population.

The strategy within selection process used to represent the binary and real coding remains the same for both optimizations. In the next sections, we will focus on the selection operator implemented in NSGA II (Deb 2007; Deb et al. 2002) and the technical coding for the parameter within the design domain. Modifications used in the above program will be also described.

\subsection{Selection function}

In the case of single objective optimization, the selection operator works as follows: after creating randomly the first generation and evaluating the objective function for all individuals, we applied the process of selection on the population. There exist a number of selection operators in the literature of genetic algorithms. The main idea consists in choosing within a population $P$ an $N$-number of individuals (to create a mating pool) that are well adapted to survive, to follow their evolution and to access to the next generation. The choice of keeping one individual is made by comparing its fitness function. One characteristic of the selection process is the selective pressure applied on the population. If this pressure is too strong, we loose the diversity within the population. If this pressure is too small, the genetic algorithm behaves randomly like Monte Carlo method. The individual with the greatest fitness value wins the tournament and is saved for the next generation. This operation (in the case of monoobjective optimization) is repeated until we obtain individuals for the next generation. It is entirely possible that some individuals participate in several tournaments: if they earn several times, they will therefore be copied several times. In this approach, the selection is strong enough. That is why we often reduce this pressure by decreasing the probability of selection in the tournament.

In the case of multiobjective optimization, the aim is to find the vector of design variables $\boldsymbol{x}=\left[x_{1}, x_{2}, x_{3}, \ldots, x_{n}\right]^{T}$ that minimizes a vector of objective functions given below.

$$
\begin{aligned}
& \operatorname{Min} F(x)=\left(f_{1}, f_{2}, \ldots, f_{k}\right) \\
& \text { subject to } \quad g_{i}(x) \geq 0 \quad i=1 \text { to } m
\end{aligned}
$$

The feasible domain (Fig. 3) defined by the constraints will be denoted by $\Omega$. $f_{i}$ are called criteria or objective functions and they represent the design objectives. A vector $\boldsymbol{x}^{*} \in \Omega$ is called Pareto-optimal solution if there is no vector $\boldsymbol{x} \in \Omega$ which would decrease some criteria without causing a simultaneous increase of at least one criterion function. 
Fig. 3 Feasible domain in the case of bi-function $f_{1}$ and $f_{2}$

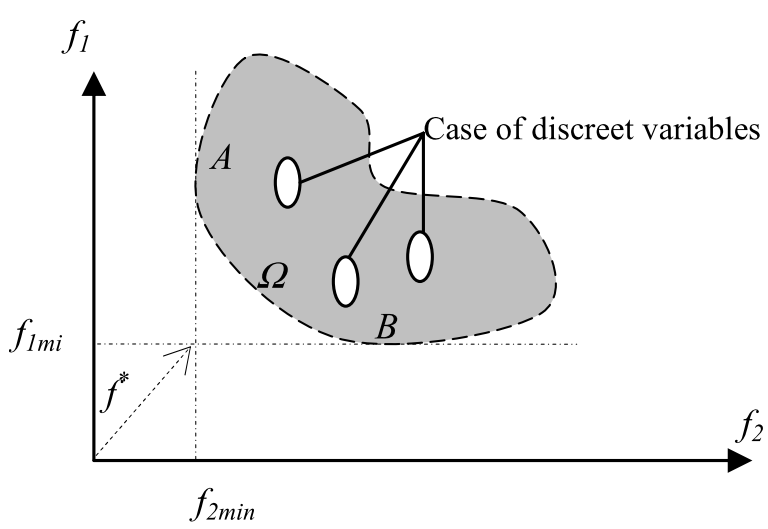

When we have mixed variables, the feasible domain includes holes or doted areas that represent the discrete coding. In this case, the selection operator changes the strategy and acts as follows: the comparison between two solutions can be achieved easily by applying the non-dominated sorting idea introduced by Deb in the NSGA code (Deb 2007; Deb et al. 2002). To continue the iterative process, the principle of choosing the solution between two solutions $\mathbf{i}$ and $\mathbf{j}$ is based on the following tree:

- Between two admissible solutions $\mathbf{i}$ and $\mathbf{j}$, we choose the one with the best objective function.

- If solution $\mathbf{i}$ is admissible and $\mathbf{j}$ inadmissible, we choose the solution $\mathbf{i}$.

- If both solutions $\mathbf{i}$ and $\mathbf{j}$ are inadmissible, we choose the one with minimum constraint violation.

Once we obtain the new generation and the new Pareto fronts in the case of multiobjective optimization, we apply the crossover and the mutation operators. With this approach, we are not sure to be located within the feasible domain $\Omega$. Other operators such as crossover and mutation remain the same as for single objective optimization. However, in the case of mixed variables, we are no longer sure to generate a feasible solution (created from two initial individuals called parents). In the next section, we will focus on the reason why two admissible solutions can lead to an infeasible solution.

\subsection{Coding of the variables}

Binary representation is often used for coding in genetic algorithms. This coding is based on $0 / 1$ representation in cells that is composed of a long string (individual) as described in Fig. 4. For instance, assume that parameter $x_{i}$ varies between $x_{i \text { min }}=0$ and $x_{i \max }=31$. The length needed for the coding is of 5 cells ( 00000 for 0 and 11111 for 31 ).

For each random representation within the variation rang, we can get the real representation of one real variable as follows:

$$
x=x+\frac{x_{\max }-x_{\min }}{2^{l}-1} \operatorname{Decode}\left(S_{x}\right)
$$


Fig. 4 Variable coding in string representation (3 parameters of 5 cells represent one individual)

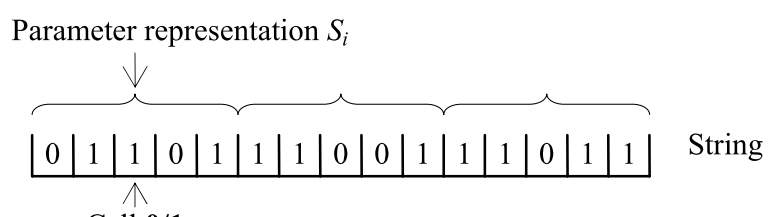

Cell 0/1

$\operatorname{Decode}\left(S_{x}\right)$ represents the integer value that comes from the $0 / 1$ representation and $l$ represents the string length used to code the variable $x$ ( $l=5$ in our case).

However, if the variation range is between 0 and 29, we will need also 5 cells to represent the parameter. In this case, the random function may exceed 29 if we consider the coding 11111 . Here, the idea is that we can either create a new random function that will be called in the case of combinatory search or use the initial random function (already implemented) to create the real parameter and to consider the around representation (Round function). The first idea became quickly obvious as during the crossover operator from two admissible solutions the crossover can produce two inadmissible solutions. The last idea seems easy to implement as it needs only the Ceil function that exists in almost all the programming languages as $\mathrm{C} / \mathrm{C}++$ for instance. The pseudo code of the round function is as follows:

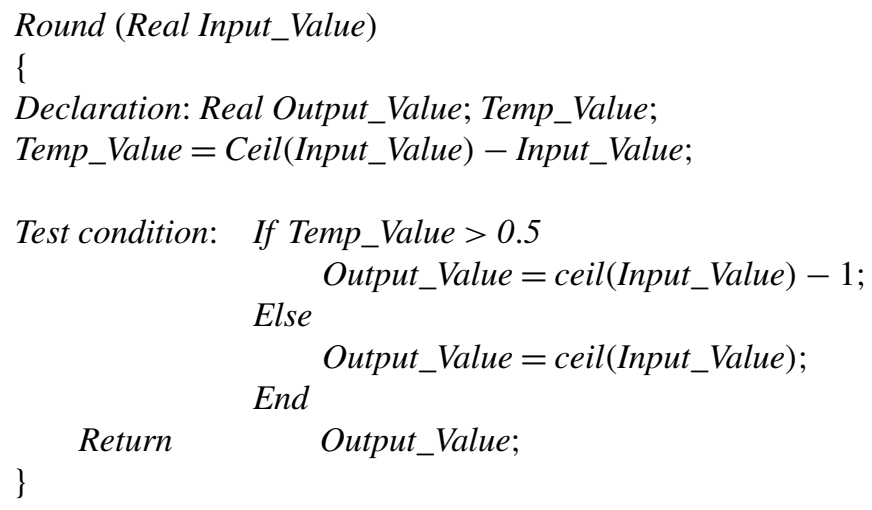

So even after the crossover, the decoding of the new string will remain within the variation rang. In NSGA-II used for the multiobjective optimization the same strategy is also implemented efficiently. In the case of real coding, we do not need to apply any other modification.

\subsection{Constraints handling}

To handle the constraints in the GA program, we consider the following composed fitness function:

$$
F(x)=\left\{\begin{array}{l}
f(x) \\
f_{\max }+C V(x) \text { if constraints violated }
\end{array}\right.
$$


$F(x)=f(x)$ if $x$ is feasible, otherwise $F(x)=f_{\max }+C V(x)$ where $f_{\max }$ is the objective function value of the most infeasible solution in the population and $C V(x)$ is the overall normalized constraint violation of the solution $x$.

Thus, there is no need to have any penalty parameter for handling the constraints as usually used in the common approaches. Constraints are normalized to avoid the scaling problems.

\section{Numerical experiments}

In this section, we start the numerical applications by a multiobjective benchmark function (convex and discontinuous) often called ZDT3 test function. Then, after the validation of our strategy, we test it on one square plate with a hole under traction and thermal loading, then on a multilayer beam fixed in one side and supported by an elastic spring at the other end.

\subsection{Benchmark function with mixed variables in multiobjective optimization}

The function ZDT3 is as follows:

$$
\begin{aligned}
& \operatorname{Min} \quad F_{1}(x)=x_{1} \\
& F_{2}(x)=g(x)\left[1-\sqrt{\frac{x_{1}}{g(x)}}-\frac{x_{1}}{g(x)} \sin \left(10 \pi x_{1}\right)\right] \\
& \text { with } g(x)=1+9 \frac{\sum_{i=2}^{n} x_{i}}{n-1}
\end{aligned}
$$

In our case, the number of variables is assumed to be $n=30$. Two cases are included: in the first case, we suppose that the entire variables vary continuously within the same rang $[0,10]$ and in the second case, the $x_{1}$ will be discrete within range $[0,10]$. For this benchmark the original rang was $[0,1]$ which gives the Pareto Front described in Fig. 5. The size of the population is 100 that evaluates along 200 generations. The probability of the crossover is equal to 0.9 and the probability of mutation is equal to 0.033 .

As the function $F_{1}$ is equal to $x_{1}$, the discrete value will be more notable on $F_{1}$ than on $F_{2}$ as shown in Fig. 7. However, along $F_{2}$ we have the continuous variation that is more linked to variables of $x_{2}$ to $x_{30}$. So, the Pareto front reduced quickly from the original curve in Fig. 6 . At the convergence, only integer points along $F_{1}$ are plotted and correspond to the Pareto solution.

In the following we will apply this strategy to a $2 \mathrm{D}$ structure under coupled load and mixed variables.

3.2 Shape, sizing and material selection optimization for the square plate with a central hole

The first optimization problem is defined over a square plate with a central hole. Usually, the plate is manufactured with mono-material, uniform thickness and a circular 

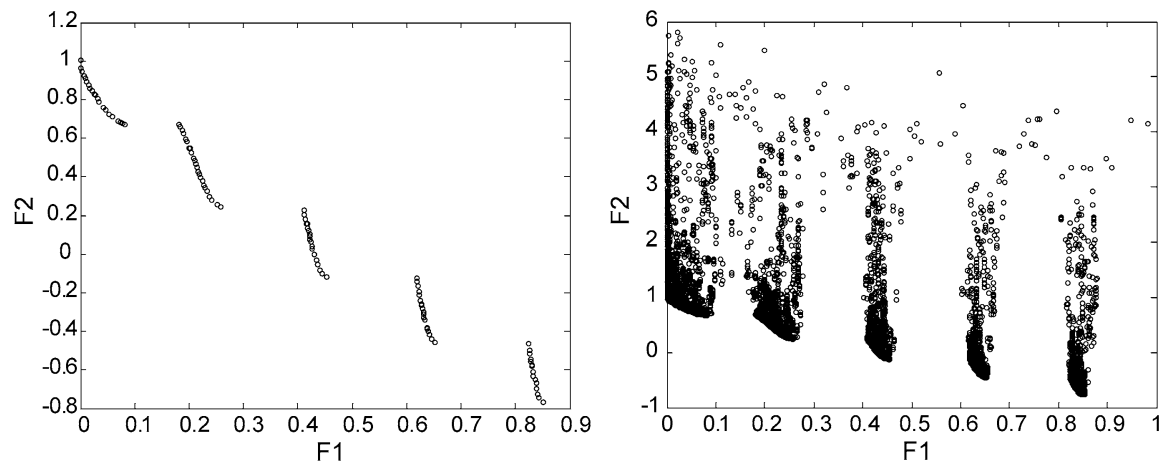

Fig. 5 Pareto front at the convergence (left) and all the populations during the entire optimization process (right) with continuous variables within rang $[0,1]$
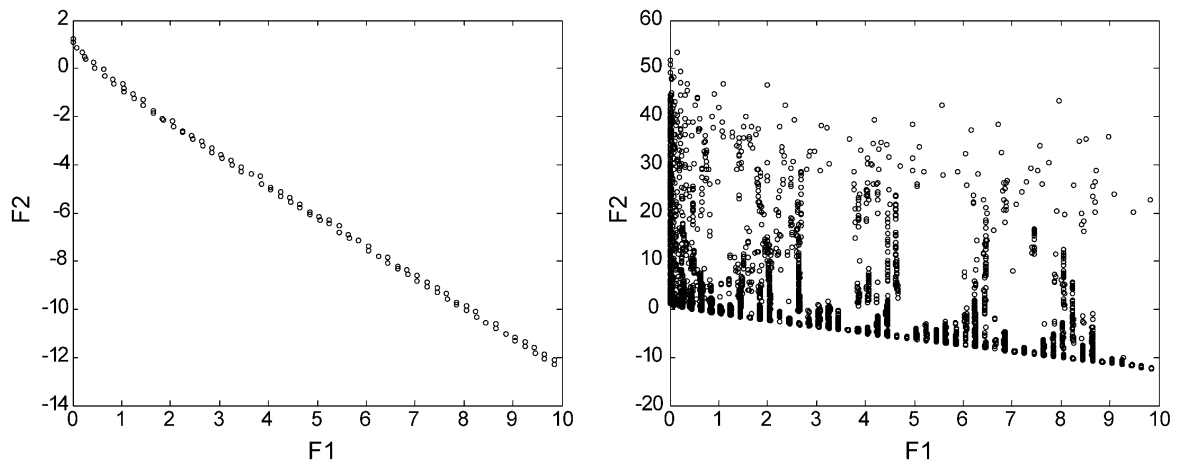

Fig. 6 Pareto front at the convergence (left) and all the population during the entire optimization process (right) with continuous variables within rang $[0,10]$

hole in the centre. For usual applications, the experiential design works well. However, the experiential design is conservative and fails to achieve a good performance when the plate endures a more complicated load, such as thermal and static loads. Selecting proper materials for different areas and designing the size or the shape of the geometry with a certain optimization method is the advisable way to approach the optimal design. Due to the double symmetry axes of the model, only a quarter of the plate has been modelled. Figure 8 illustrates the geometrical model of the plate. The plate is placed in a non-uniform temperature field which is decreasing linearly from the centre to the outside. For the sake of simplification, we construct the plate with four square sub-areas $A_{i}(i=1,2,3,4)$. For the $i$ th sub-area $A_{i}(i=1,2,3,4)$, a temperature $T_{i}(i=1,2,3,4)$ and a material $m_{i}(i=1,2,3,4)$ are specified. The temperature decreases from the centre with a constant step $\Delta T$ for each sub-area, which can be formulized as $T_{i+1}=T_{i}-\Delta T(i=1,2,3)$. Usually, a material library with a total material number of $N$ is predefined for the material selection procedure. Materials can only be chosen from the material library. For the first example, a material library containing $N=6$ different materials is used, which is listed with Table 1. 

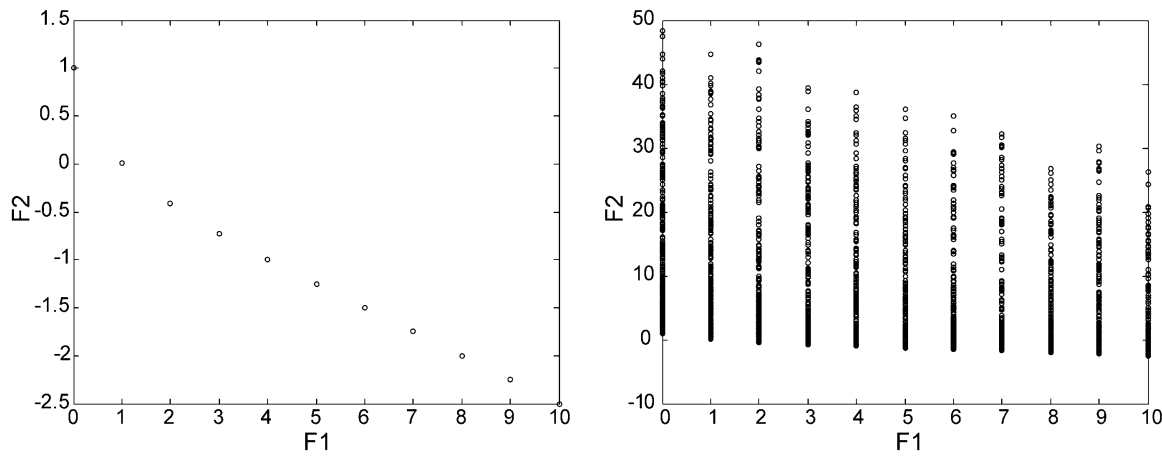

Fig. 7 Pareto front at the convergence (left) and all the population during the entire optimization process (right) with mixed variables within the rang $[0,10]$
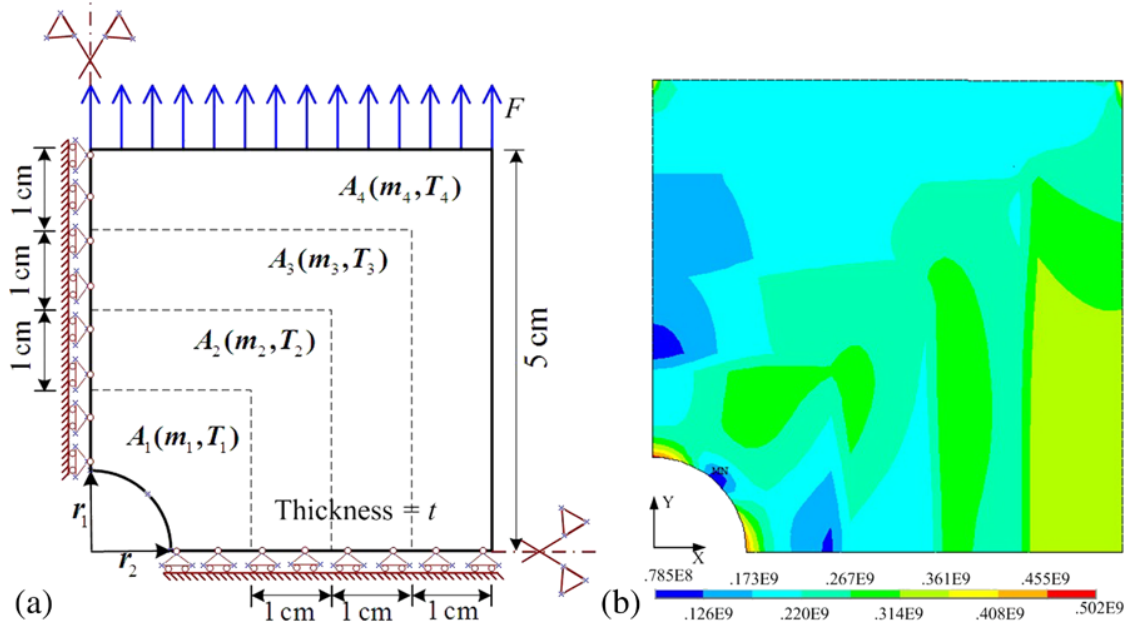

Fig. 8 (a) Square plate with a central hole; (b) stresses contours of original design

The shape of the central hole is defined by two parameters $r_{1}, r_{2}$. When $r_{1}=r_{2}$, the central hole is circular. Otherwise, the central hole is elliptical. The plate is modelled with uniform thickness $t$, which is also considered as a design variable in the following examples.

Because of the non-uniformity of the temperature, the distribution of the material will significantly influence the distortion and the stress concentration of the structure. Besides the temperature load, a uniform tension $F$ is also applied on two opposite edges of the plate. Consequently, the basic objective of the optimization problem is to minimize the maximum stress $\left(\sigma_{\max }\right)$ which occurs near the hole or the interface of two sections. The minimum value of $\sigma_{\max }$ can be achieved by optimally selecting the material for each component in the structure. At the same time, the total weight of the plane is taken into account as well. The problem is a multiobjective optimization considering the minimization of both the maximum stress and the total weight. The material IDs $m_{i}(i=1,2,3,4)$ for the $i$ th sub-area are treated as binary design vari- 
Table 1 Material library with 6 materials

\begin{tabular}{llllll}
\hline Material ID $\left(m_{i}\right)$ & Name & $E(\mathrm{GPa})$ & $\mu$ & $\alpha\left(10^{-6} /{ }^{\circ} \mathrm{C}\right)$ & $\rho\left(10^{3} \mathrm{~kg} / \mathrm{m}^{3}\right)$ \\
\hline 1 & Alloy 1 & 185 & 0.32 & 13.8 & 8.44 \\
2 & Alloy 2 & 178 & 0.29 & 13.31 & 8.89 \\
3 & Alloy 3 & 164 & 0.32 & 13.08 & 8.27 \\
4 & Aluminum & 70 & 0.33 & 23 & 2.6 \\
5 & Copper & 100 & 0.34 & 20 & 8.5 \\
6 & Steel & 200 & 0.27 & 15 & 7.85 \\
\hline
\end{tabular}
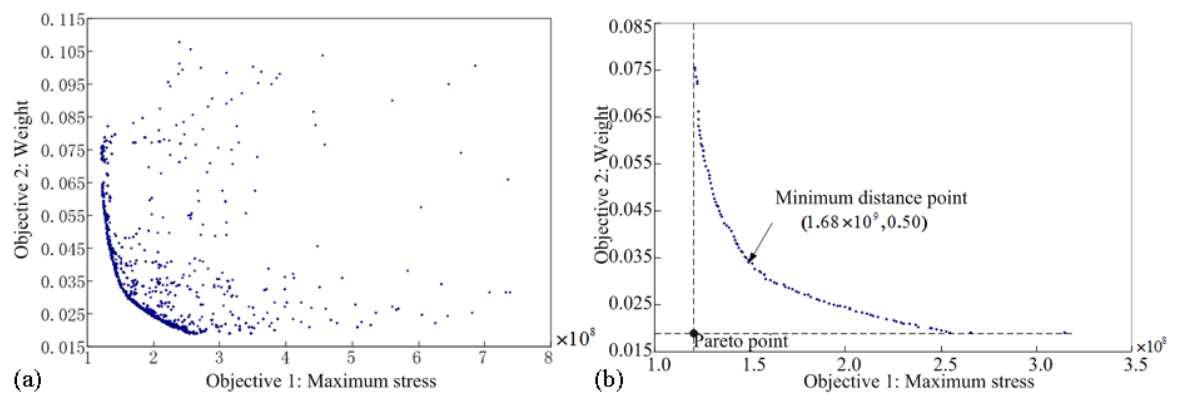

Fig. 9 (a) All the population during the optimization process and (b) Pareto optimal front at the convergence

ables, while the parameters $r_{1}, r_{2}$ and $t$ are real design variables. The optimization problem is then formulized as follows:

$$
\begin{array}{ll}
\text { find: } & \left\{m_{1}, m_{2}, m_{3}, m_{4} ; r_{1}, r_{2}, t\right\} \\
\text { min: } \quad & \left\{\sigma_{\max }, W\right\} \\
\text { s.t. } & \left\{\begin{array}{l}
m_{i} \in\{1,2,3,4,5,6\} \quad(i=1,2,3,4) \\
0.5 \mathrm{~cm} \leq r_{1}, r_{2} \leq 1.5 \mathrm{~cm} \\
0.1 \mathrm{~cm} \leq t \leq 0.5 \mathrm{~cm}
\end{array}\right.
\end{array}
$$

where $W$ denotes the weight of the plate.

The model is originally initialized as uniform material distribution with $m_{1}=$ $m_{2}=m_{3}=m_{4}=1$. The temperatures of the structure are defined as: $T_{1}=600 \mathrm{~K}$, $\Delta T=50 \mathrm{~K}, T_{\mathrm{Ref}}=300 \mathrm{~K}$. The central hole is circular with the following radius $r_{1}=r_{2}=1 \mathrm{~cm}$. The thickness of the plate is defined with $t=0.1 \mathrm{~cm}$. The stresses distribution of the original design is illustrated in Fig. 8b. We can see from the stresses contours that the stress concentration phenomena take place on the edge of the hole. In this example, we had not included any additional constraints accepted the ones of the variables. In Fig. 9a, all the population during the entire optimization process is plotted. The Pareto front at the convergence (Fig. 9b) shows also the convexity of the defined problem which is the case in general of such structural examples when we consider the following functions: stress and weight. 
(a)
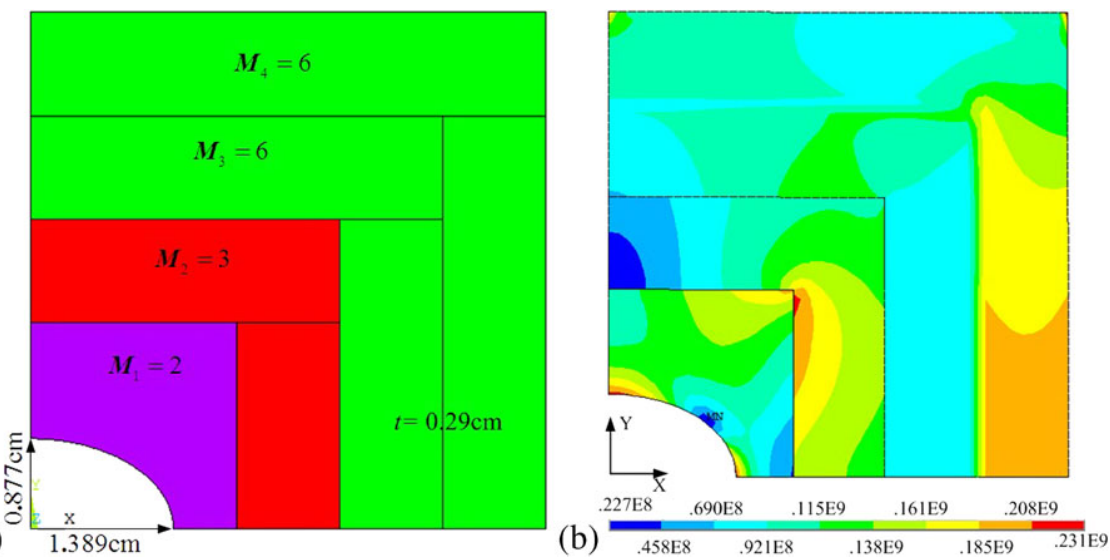

Fig. 10 (a) The final design of the plate (left) and (b) the associated stress result (right)

From the unfeasible Pareto optimal point front illustrated in Fig. 9b, we can obtain one feasible Pareto point at the convergence. This point represents the nearest point in terms of metrics to the unfeasible Pareto point. It is noted that, the two objective functions should be normalized to $0 \sim 1$ to eliminate the effect of quantitative scale. The final design of the model is illustrated in Fig. 10a. The optimal material assignment for each sub-area is $m_{1}=2, m_{2}=3, m_{3}=6$ and $m_{4}=6$; the optimal parameters for the central hole is $r_{1}=0.877 \mathrm{~cm}$ and $r_{2}=1.389 \mathrm{~cm}$ which represents an elliptical shape. The stresses distribution of the final design is illustrated in Fig. 10b. From the stresses contours, we can see that the stress concentration phenomena transfer from the edge of the hole in the original design to the interface of different areas in the final design with an obvious quantitative decrease. The shape reconfiguration of the central hole and the reassignment of the material selection make significant contribution to improving the structural performance of the plate.

3.3 Sizing and material selection optimization of a multilayer beam supported by springs

The second example concerns simultaneously sizing, material and spring optimization of a multilayer beam. The beam contains five layers of different materials. One end of the beam is clamped and the other one is supported by two elastic springs. Suppose the beam is loaded by a concentrated force in the centre of the upper surface as well as a uniform temperature load on the whole body. Since each layer is probably assigned with a different material, the stress concentration may take place in the interfaces of different layers. A material selection approach is carried out to optimize mechanical and thermal performances of the beam by minimizing the maximum stress. Meanwhile, the weight of the beam is reduced. Thus, the minimization of the weight is the other objective function. A material ID $m_{i}(i=1,2, \ldots, 5)$ is considered as integer design variable for each layer. Meanwhile, the thickness of each layer 


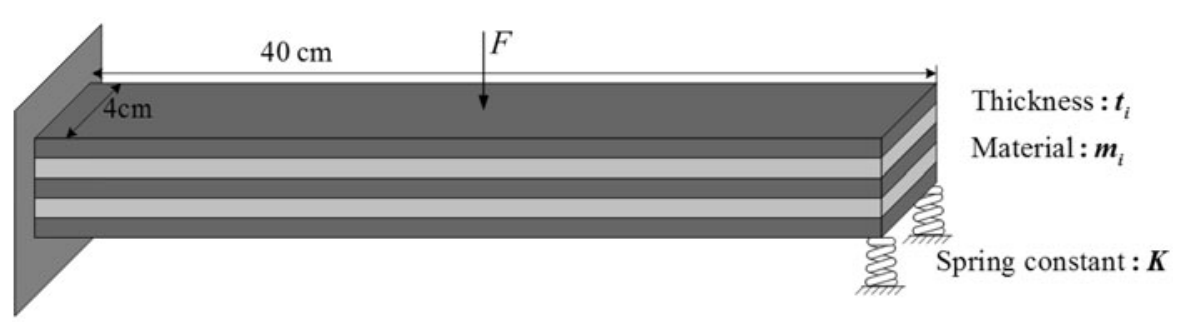

Fig. 11 Multilayer beam

Table 2 Material library with 16 materials

\begin{tabular}{lllll}
\hline Material ID $\left(m_{i}\right)$ & $E(\mathrm{GPa})$ & $\mu$ & $\alpha\left(10^{-6} /{ }^{\circ} \mathrm{C}\right)$ & $\rho\left(10^{3} \mathrm{~kg} / \mathrm{m}^{3}\right)$ \\
\hline 1 & 185 & 0.32 & 13.8 & 8.44 \\
2 & 178 & 0.29 & 13.31 & 8.89 \\
3 & 164 & 0.32 & 13.08 & 8.27 \\
4 & 160 & 0.32 & 14.4 & 8.24 \\
5 & 137 & 0.3 & 15.5 & 8.4 \\
6 & 135 & 0.3 & 17.3 & 7.93 \\
7 & 73 & 0.33 & 24.3 & 2.7 \\
8 & 73 & 0.33 & 22.7 & 2.77 \\
9 & 117 & 0.30 & 16.6 & 8.9 \\
10 & 44.8 & 0.35 & 26.1 & 1.77 \\
11 & 196 & 0.3 & 12.9 & 7.4 \\
12 & 221 & 0.30 & 13 & 8.9 \\
13 & 193 & 0.29 & 17.8 & 8.03 \\
14 & 102 & 0.3 & 9.36 & 4.58 \\
15 & 71.7 & 0.33 & 24.7 & 2.74 \\
16 & 207 & 0.29 & 15.1 & 7.85 \\
\hline
\end{tabular}

$t_{i}(i=1,2, \ldots, 5)$ and the elastic stiffness $K$ of the two supporting springs are also considered as continuous design variables. In our example, the beam is specified to be five-layered, and a more detailed material library containing 16 metal or alloy materials is predefined for the material selection as shown in Table 2. The design model of the problem is illustrated in Fig. 11.

The optimization procedure is carried out at two stages. At the first stage, displacements of the structure are not taken into account. So the multiobjective optimization problem contains no constraints. At the second stage, the maximum displacement is considered as a constraint. Both kinds of multiobjective optimizations are formulated as: 
Fig. 12 Pareto optimal front at the convergence for the first problem

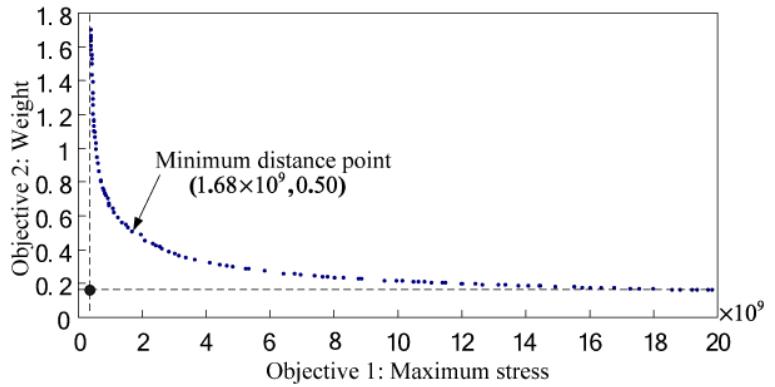

Problem 1 Multiobjective optimization without constraint

find: $\quad\left\{m_{1}, m_{2}, \ldots, m_{5} ; t_{1}, t_{2}, \ldots, t_{5}, K\right\}$

$\min : \quad\left\{\sigma_{\max }, W\right\}$

s.t. $\begin{cases}m_{i} \in\{1,2, \ldots, 16\} & (i=1,2, \ldots, 5) \\ t_{\min } \leq t_{i} \leq t_{\max } & (i=1,2, \ldots, 5) \\ K_{\min } \leq K \leq K_{\max } & \end{cases}$

Problem 2 Multiobjective optimization with constraint on displacements

$$
\begin{aligned}
& \text { find: } \quad\left\{m_{1}, m_{2}, \ldots, m_{5} ; t_{1}, t_{2}, \ldots, t_{5}, K\right\} \\
& \text { min: } \quad\left\{\sigma_{\max }, W\right\} \\
& \text { s.t. } \begin{cases}u_{\max } \leq u_{0} \\
m_{i} \in\{1,2, \ldots, 16\} & (i=1,2, \ldots, 5) \\
t_{\min } \leq t_{i} \leq t_{\max } & (i=1,2, \ldots, 5) \\
K_{\min } \leq K \leq K_{\max }\end{cases}
\end{aligned}
$$

where, $m_{i}, t_{i}$ denote the material ID and the thickness of the $i$ th layer, respectively. The two objectives, $\sigma_{\max }$ and $W$, are the maximum equivalent stress and the beam weight. $u_{\text {max }}$ denotes the maximum displacement, while $K$ denotes the spring constant. The upper bound $u_{0}$ is assigned as $5 \mathrm{~cm}$. In both formulations, the bounds of $t_{i}$ are defined as $[0.1,0.5](\mathrm{cm})$, while the bounds of $K$ are defined as $\left[2 \times 10^{4}, 2 \times 10^{5}\right]$ $(\mathrm{N} / \mathrm{m})$.

Note that the Pareto optimal front has a convex curve in Fig. 12. However, the maximum stress function dominates often the design process when compared with the weight function. In the case, when we introduce constraints on displacement, the over domination of the maximum stress function decreases quickly as the importance of the weight increases with displacement effects as illustrated in Figs. 12 and 13, respectively. Most practical optimization problems include several constraints. Sometimes, we ignore or we increase the importance of some constraints to have an overview of the design process. Constraints on displacement are also introduced to get more practical and reasonable results. From the comparison of the Pareto optimal front of the two problems (with and without constraints) illustrated in Fig. 13, we can 
Fig. 13 Pareto optimal front at the convergence for the second problem

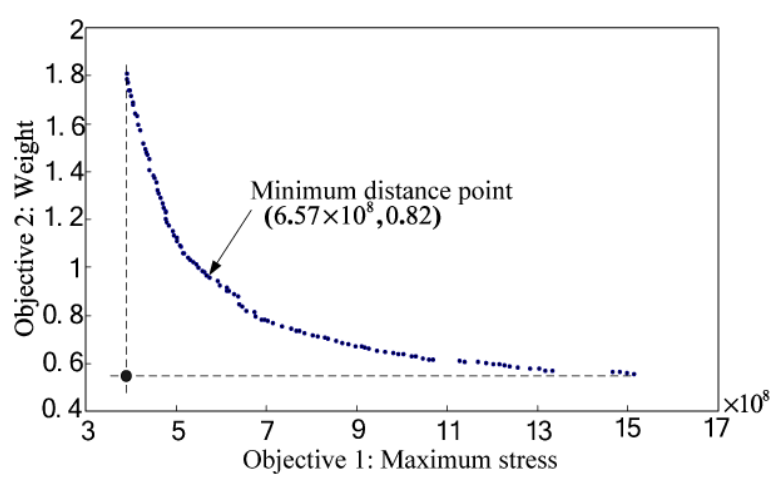

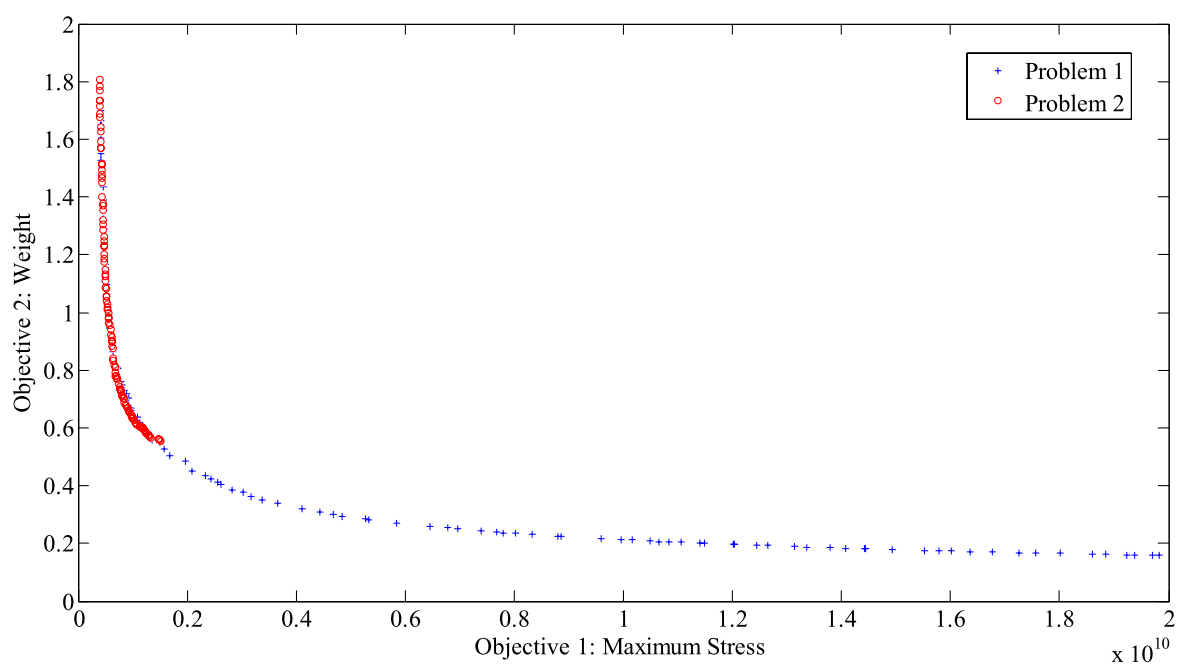

Fig. 14 Representation of the Pareto optimal front for problems 1 and 2

note that the Pareto optimal front of optimization problem with constraints is just a subset of the Pareto optimal front in the optimization problem without constraints. The Pareto point and the minimum distance point for both problems are figured out in Fig. 13. In terms of minimum distance between the unfeasible Pareto point and the feasible Pareto front, the value changes slightly. The optimal design in terms of minimum distance point for the optimization problem with constraint on displacement is illustrated in Fig. 15 with the detailed results listed in Table 3.

\section{Conclusions}

This article is concerned with simultaneous designs of material selection and geometry optimization under static and thermal loads. Most structural designers have to design with materials that are already available in the market. This need had become realistic with the described strategy based on a modified genetic algorithm. 
Fig. 15 The optimal design in the case of the optimization problem with constraint on displacements

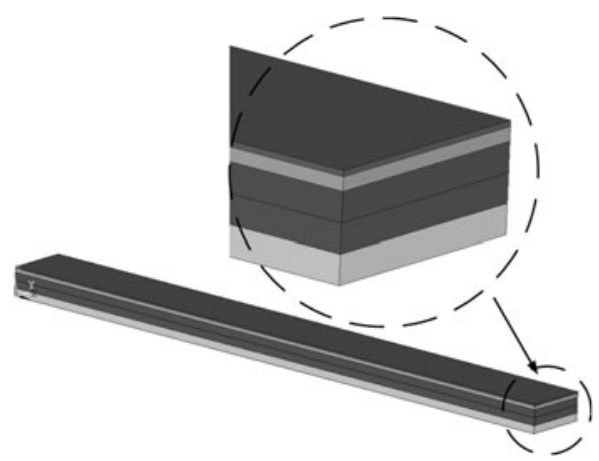

Table 3 Detailed result list associated with Fig. 15

\begin{tabular}{llllll}
\hline Design variables & & & & Objective functions & \\
\cline { 1 - 1 } \cline { 5 - 6 } Thickness $(\mathrm{m})$ & Material ID & Spring constant $(\mathrm{N} / \mathrm{m})$ & & Maximum stress $\left(\mathrm{N} / \mathrm{m}^{2}\right)$ & Weight $(\mathrm{kg})$ \\
\hline 0.004959 & 15 & & 656651968 & 0.816736 \\
0.004871 & 10 & & & \\
0.004987 & 10 & 97834.625000 & & \\
0.002321 & 16 & & & \\
0.001010 & 10 & & & \\
\hline
\end{tabular}

Materials are selected from a database defined by an index to choose the best combination with the corresponding structure parameters (sizing and shape design). This mixed optimization considers either continuous or discreet parameter for the optimization problem. Different numerical examples are presented to illustrate the influence of the discrete choice within a continuous range or a database.

The originality of this work come out on the handling of the mixed parameters in the case of the multi-objective optimization with the difficulties we can face related to convexity and the continuity of the Pareto domain.

As perspective of the approach introduced in this article, it can be easily extended to more complicated problems that consider not only sizing and shape optimization but also topology optimization.

Open Access This article is distributed under the terms of the Creative Commons Attribution Noncommercial License which permits any noncommercial use, distribution, and reproduction in any medium, provided the original author(s) and source are credited.

\section{References}

Alexopoulos ND (2007) Generation of quality maps to support material selection by exploiting the quality indices concept of cast aluminum alloys. Mater Des 28(2):534-543

Bassir DH, Zapico VJL, González MP (2007) Identification of spatial linear model based on earthquakeinduced data and genetic algorithm with parallel selection. Int J Simul Multidiscip Des Optim 1:39_ 48 
Bassir DH, Abdella M, Gurdal Z (2009a) New sorting approach for Pareto fronts in multiobjectives optimization. 50th AIAA/ASME/ASCE/AHS/ASC structures, structural dynamics, and materials conference, Palm Springs, CA, USA

Bassir DH, Guessasma S, Boubakar ML (2009b) Hybrid computational strategy based on ANN and GAPS: Application for identification of a non linear composite material. J Compos Struct 88(2):262-270

Bendsoe M et al (1994) An analytical model to predict optimal material properties in the context of optimal structural design. J Appl Mech 61(4):930-937

Deb K (2007) Current trends in evolutionary multi-objective optimization. Int J Simul Multidiscip Des Optim 1:1-8

Deb K et al (2002) A fast and elitist multi-objective genetic algorithm: NSGA-II. IEEE Trans Evol Comput 6(2):181-197

Deng YM, Edwards KL (2007) The role of materials identification and selection in engineering design. Mater Des 28(1):131-139

Edwards KL (2005) Selecting materials for optimum use in engineering components. Mater Des 26(5):469-473

Edwards KL, Deng YM (2007) Supporting design decision-making when applying materials in combination. Mater Des 28(4):1288-1297

Fredricson H (2005) Topology optimization of frame structures-joint penalty and material selection. Struct Multidiscip Optim 30(3):193-200

Ghoreishi SR et al (2007) Analytical modeling of synthetic fiber ropes subjected to axial loads. Part I: A new continuum model for multilayered fibrous structures. Int J Solids Struct 44(9):2924-2942

Goldberg D (1989) Genetic algorithms in search, optimisation, and machine learning. Addison-Wesley, New York

Guessasma S, Bassir DH (2010a) Optimisation of mechanical properties of virtual porous solids using a hybrid approach. Acta Mater 58:716-725

Guessasma S, Bassir DH (2010b) Identification of mechanical properties of biopolymer composites sensitive to interface effect using hybrid approach. Mech Mater 42:344-353

Guisbiers G, Wautelet M (2007) Materials selection for micro-electromechanical systems. Mater Des 28(1):246-248

Holland JH (1975) Adaptation in natural and artificial systems. University of Michigan Press, Ann Arbor

Hörnlein HREM, Kocvara RWM (2001) Material optimization: bridging the gap between conceptual and preliminary design. Aerosp Sci Technol 5(8):541-554

Irisarri FX, Bassir DH, Maire JF, Carrere N (2009) Multiobjective stacking sequence optimisation strategy for laminated composite structures. Compos Sci Tech 69:983-990

Jung D, Gea H (2006) Design of an energy-absorbing structure using topology optimization with a multimaterial model. Struct Multidiscip Optim 32(3):251-257

Kumar S, Singh R (2007) A short note on an intelligent system for selection of materials for progressive die components. J Mater Process Technol 182(1-3):456-461

Matos MJ, Simplio MH (2006) Innovation and sustainability in mechanical design through materials selection. Mater Des 27(1):74-78

Michalewicz Z (1994) Genetic algorithms + data structures = evolution. Springer, Heidelberg

Rakshit S, Ananthasuresh G (2008) Simultaneous material selection and geometry design of statically determinate trusses using continuous optimization. Struct Multidiscip Optim 35(1):55-68

Rao RV (2006) A material selection model using graph theory and matrix approach. Mater Sci Eng A 431(1-2):248-255

Tawancy HM et al (2007) Effect of materials selection and design on the performance of an engineering product-An example from petrochemical industry. Mater Des 28(2):686-703

Torstenfelt B, Klarbring A (2007) Conceptual optimal design of modular car product families using simultaneous size, shape and topology optimization. Finite Elem Anal Des 43(14):1050-1061

Walker M, Smith R (2002) A computational methodology to select the best material combinations and optimally design composite sandwich panels for minimum cost. Comput Struct 80(18-19):14571460

Walker M, Smith R (2006) A procedure to select the best material combinations and optimally design composite sandwich cylindrical shells for minimum mass. Mater Des 27(2):160-165

Walker M, Reiss T, Adali S (1997) A procedure to select the best material combinations and optimally design hybrid composite plates for minimum weight and cost. Eng Opt 29:65-83 
Zhang WH, Domaszewski M, Bassir DH (1999) Developments of sizing sensitivity analysis with ABAQUS code. J Struct Optim 17:219-225

Zhang WH et al (2007) Using strain energy-based prediction of effective elastic properties in topology optimization of material microstructures. J Acta Mech Sin 23(1):77-89

Zhang WH et al (2008) A new algorithm for the numerical simulation of machined surface topography in multiaxis ball-end milling. J Manuf Sci Eng 130:011003 